\title{
Patient centered imaging and the dose of radiopharmaceuticals
}

\author{
Randall C. Thompson, MD, FASNC, ${ }^{a}$ and Eric V. Burgett, CNMT, NCT ${ }^{\mathrm{a}}$ \\ a St. Luke's Mid America Heart Institute, Kansas City, MO
}

Received Aug 5, 2015; accepted Aug 5, 2015

doi: $10.1007 / \mathrm{s} 12350-015-0263-8$

\section{See related article, pp. 134-142}

The importance of tailoring imaging to the patient has been a significant focus of the medical imaging community, and particularly the American Society of Nuclear Cardiology in recent years. ${ }^{1}-{ }^{4}$ The key principals of this desirable individualized approach include justification, that is, appropriate testing, and optimization, i.e., performing the test in an ideal manner. This latter principal includes issues of convenience and comfort to the patient, cost, and especially image quality and radiation dose.

In this issue of the Journal, the study by van Dijk and colleagues provides useful data to guide individualized dosing of radiopharmaceutical for stress first myocardial perfusion imaging (MPI). ${ }^{5}$ These data help document when a lower than usual dose can be administered and when a higher than standard dose is justified for proper image quality. In other words, the authors help move us in the direction of patient centered imaging and optimizing the most common of nuclear cardiology tests.

van Dijk and colleagues present us with a formula for dose adjustment that includes body weight and scan time. This publication is useful. Previous empiric data regarding optimized technique for Anger cameras are surprisingly lacking. The approach taken by van Dijk and colleagues to derive their data utilized a fixed acquisition time on a standard Anger camera, and their example table displays increasing radioisotope dose

Reprint requests: Randall C. Thompson, MD, FASNC, St. Luke's Mid America Heart Institute, 4330 Wornall Rd, Suite 2000, Kansas City, MO 64111; rthompson@saint-lukes.org

J Nucl Cardiol 2016;23:143-4.

$1071-3581 / \$ 34.00$

Copyright (C) 2015 American Society of Nuclear Cardiology. according to body weight assuming a 10-minute acquisition.

As an alternative to the approach of adjusting the dose of the radioisotope, one can instead adjust the length of the acquisition time and keep the dose relatively constant. This latter approach is sometimes challenging to do with Anger cameras, but is commonly employed with the newer technology high-resolution cadmium-zinc-telluride (CZT) cameras. The CZT instruments can allow one to determine cardiac counts during the scanning, so the length of the acquisition can be adjusted to obtain a target number of counts. Our laboratory, for example, routinely administers $4 \mathrm{mCi}$ of Tc $99 \mathrm{~m}$ for stress first MPI studies on CZT cameras and adjusts the scan time to acquire 1 million counts. This acquisition time is about 14 minutes.

Since Anger cameras do not typically allow one to determine the cardiac counts during scanning, fixed protocols or adjustment protocol formulas such as the one offered by van Dijk and colleagues need to be employed. At our institution, we use a relatively fixed dose and relatively fixed acquisition time for stress first, 1 day studies on Anger cameras. Patients receive approximately $9 \mathrm{mCi}$ of Tc $99 \mathrm{~m}$ (range 7-12 $\mathrm{mCi}$ ) and image acquisition time is roughly 22 minutes for dual headed cameras. Attenuation correction and wide beam reconstruction are routinely used. In fact, the acquisition time could often be shortened, except for the duration needed to obtain a quality line source attenuation map and to have enough counts per bin for 16 frames per cardiac cycle gating. Patients who have a BMI $>35$ are tested with different protocols, such as 2 day or rest first. The protocol tested by van Dijk and colleagues used a short fixed acquisition time of 10 minute. This short acquisition time might translate into laboratory efficiency and lower cost. If it does not, the dose of radiation should be reduced further. Based on the van Dijk formula, an acquisition time of 20 minute would theoretically allow one to reduce the dose of Tc $99 \mathrm{~m}$ to 5-8 $\mathrm{mCi}$. 
Table 1. Comparison of Tc $99 \mathrm{~m}$ dose proposed for stress first MPI imaging on an Anger camera by van Dijk et al. ${ }^{5}$ to the actual dose administered for stress first imaging at the Mid America Heart Institute 2011-2015

\begin{tabular}{|c|c|c|c|}
\hline Weight (kg) & $\begin{array}{c}\text { van Dijk et al. }{ }^{5} 10 \text { minute } \\
\text { acquisition } \\
\text { Tracer dose (mCi) }\end{array}$ & $\begin{array}{c}\text { MAHI } 2011-2015 * \approx 22 \text { minute } \\
\text { acquisition } \\
\text { Tracer dose }(\mathbf{m C i})\end{array}$ & $\mathbf{n}$ \\
\hline$<60$ & 8.6 & 9.3 & 292 \\
\hline 65 & 9.4 & 9.4 & 175 \\
\hline 70 & 9.9 & 9.4 & 232 \\
\hline 75 & 10.4 & 9.3 & 293 \\
\hline 80 & 10.9 & 9.4 & 341 \\
\hline 85 & 11.3 & 9.4 & 353 \\
\hline 90 & 11.8 & 9.4 & 342 \\
\hline 95 & 12.2 & 9.3 & 299 \\
\hline 100 & 12.7 & 9.5 & 245 \\
\hline 105 & 13.1 & 9.4 & 299 \\
\hline 110 & 13.6 & 9.5 & 214 \\
\hline 115 & 14.0 & 9.4 & 148 \\
\hline 120 & 14.4 & 9.7 & 73 \\
\hline 125 & 14.8 & 9.7 & 44 \\
\hline 130 & 15.3 & 9.6 & 19 \\
\hline$>130$ & 15.3 & 9.4 & 3 \\
\hline
\end{tabular}

* Routine attenuation correction and advanced post processing

In Table 1 above, the example radioisotope dose from van Dijk's study (with a 10 minute acquisition) is compared to the mean actual doses administered in our laboratory (with a 22 minute acquisition) during the past 5 years for stress first studies on Anger cameras. The table illustrates several points. Firstly, the administered dose of radiopharmaceutical using the current approach at our laboratory is lower for all except the lightest patients in the van Dijk example table. There is an important trade off between dose (patient safety) and scan time (patient comfort and laboratory efficiency). Secondly, in low body weight patients, the radioisotope dose can be lowered in our protocol, and probably almost all standard protocols. Thirdly, when a short scan time is used, the radioisotope dose needed in heavy patients is too high to make it practical for 1 day stressrest imaging. The same day rest dose would need to be about three times as large, exceeding recommended 1 day dosing limits for large patients. Thus, these patients need longer acquisition times if a single day, stress first protocol is to be employed.

We strongly endorse the concept of keeping the focus on the patient and of the wider use of patient-specific protocols. The study by van Dijk and colleagues provides us with a formula to consider in order to accomplish the goal of tailoring and optimizing the nuclear cardiac stress test for the particular patient being tested. The next steps are to further validate this formula and then to apply it in a manner to routinely lower radiation dosages.

\section{Disclosure}

The authors have indicated that they have no financial conflict of interest.

\section{References}

1. DePuey EG, Mahmarian JJ, Miller TD, Einstein AJ, Hansen CL, Holly TL, Miller EJ, Polk DM, Wann LS. ASNC preferred practive statement: Patient-centered imaging. J Nucl Cardiol. 2012;19:185215.

2. Einstein AJ, Berman DS, Min JK, Hendel RC, Gerber TC, Carr JJ, Cerqueira MD, Cullom SJ, DeKemp R, Dickert NW, Dorbala S, Fazel R, Garcia EV, Gibbons RJ, Halliburton SS, Hausleiter J, Heller GV, Jerome S, Lesser JR, Raff GL, Tilkemeier P, Williams KA, Shaw LJ. Patient-centered imaging: Shared decision making for cardiac imaging procedures with exposure to ionizing radiation. J Am Coll Cardiol. 2014;63:1480-9.

3. Jha S, Boonn W. Patient-centered imaging: Opportunities and challenges. J Am Coll Rad. 2012;9:157-9.

4. Stern RG. Patient centered imaging. Am J Med. 2012;125:115-7.

5. van Dijk JD, Jager PL, Ottervanger JP, de Boer J, Oostdijk AHJ, Engbers EM, Slump CH, Knollema S, van Dalen JA. Development and validation of a patient-tailored dose regime in myocardial perfusion imaging using conventional SPECT. J Nucl Cardiol. 2015. doi:10.1007/s12350-015-0246-9. 